\title{
Systemic lupus erythematosus and primary cerebral lymphoma
}

\author{
A.S. Woolf and G. Conway \\ Medical Unit, Middlesex Hospital, Mortimer Street, London W.1, UK.
}

\begin{abstract}
Summary: A woman with a 21 year old history of systemic lupus erythematosus (SLE) is presented. During a period of low connective tissue disease activity she developed sudden difficulty in walking and after much investigation was found to have a primary lymphoma of the brain. She died 6 months after this diagnosis from meningeal spread, despite radiotherapy to the tumour. The association between SLE and lymphoma is discussed.
\end{abstract}

\section{Introduction}

Impaired immunity in systemic lupus erythematosus (SLE) has many consequences. It may arise from the disease itself or be secondary to drug therapy. It may explain the increased risk of lymphoma and infection in this disease. We present a case of primary cerebral lymphoma in SLE which masqueraded briefly as toxoplasmosis.

\section{Case report}

In late 1982, a 54 year old woman with SLE for 21 years presented with sudden onset of weakness of her left leg which caused her to limp. On examination this limb showed a pyramidal type of weakness and an extensor plantar response. There was a faint erythematous rash on her face but no other sign of active connective tissue disease.

In 1964 she had developed Raynaud's phenomenon and scalp lesions that were compatible with SLE and was treated with mepacrine. She remained well until 1980 when she developed pericarditis and the nephrotic syndrome. DNA binding was 1/640 and a renal biopsy showed membranous change and mesangial proliferation. Over the next 4 years she received 7 courses of methylprednisolone $1 \mathrm{~g} \times 3$ days, with a maintenance dose of prednisolone $10 \mathrm{mg}$ on alternate days. Azathioprine ( $100 \mathrm{mg} /$ day) was added for 9 months in 1984. With this treatment her symptoms resolved, her DNA titre became negative and her urinary protein excretion became normal.

Computerized tomography (CT) scan of the brain was performed in September 1985, soon after the

Correspondence: A.S. Woolf, M.R.C.P., A9 Endocrine Unit, Archway Wing, Whittington Hospital, Highgate Hill, London N.19, UK.

Accepted: 20 January 1987 current presentation. It showed enhancing lesions in the right temporal and parietal lobes (Figure 1a). The appearance was thought to be compatible with lymphoma, toxoplasmosis or direct involvement of the brain by SLE. Cerebral arteriograms showed no abnormal circulation. Her cerebrospinal fluid (CSF) contained $0.56 \mathrm{~g} / \mathrm{l}$ of protein and showed no abnormal cells. Her toxoplasma titre on presentation was 1/1024. Although her toxoplasma IgM titre was negative she was given a trial of pyrimethamine, sulphadiazine and folinic acid. However, after one week of this therapy, her SLE flared up with a worsening rash, the reappearance of proteinuria and a fall in renal function. Her DNA titre again became positive. Therefore anti-toxoplasma therapy was stopped because of a presumed drug-induced exacerbation of her SLE.

Over the following month her weakness progressed and a stereotactic brain biopsy was performed. Histology showed a diffuse mixed follicle centre cell lymphoma (diffuse, centroblastic and centrocytic in the Kiel classification). Previous reports in the literature would include such tumours in the categories 'reticulum cell sarcoma' or 'microglioma' (Figure 2). There were no clinical signs of distant deposits and CT scans of the thorax and abdomen showed no sign of tumour elsewhere in the body. Whole brain irradiation was performed with marked CT scan resolution (Figure $1 \mathrm{~b}$ ) but unfortunately only partial clinical improvement.

In January 1986 she developed right shoulder weakness and examination showed signs of a lower motor neurone lesion. Her CSF now contained $4 \mathrm{~g} / \mathrm{l}$ of protein, less than $0.1 \mathrm{mmol} / 1$ of glucose and sheets of neoplastic cells. CSF spread of her tumour was diagnosed and intrathecal methotrexate administered. Despite this treatment her condition deteriorated and she died in March 1986. 
2

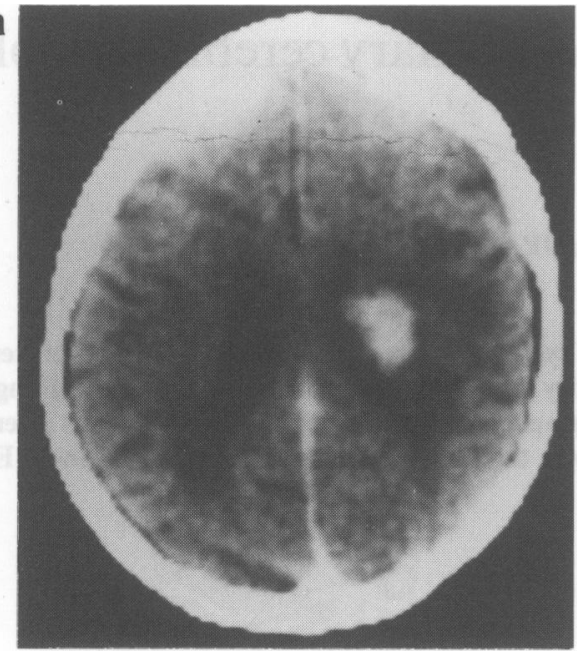

b

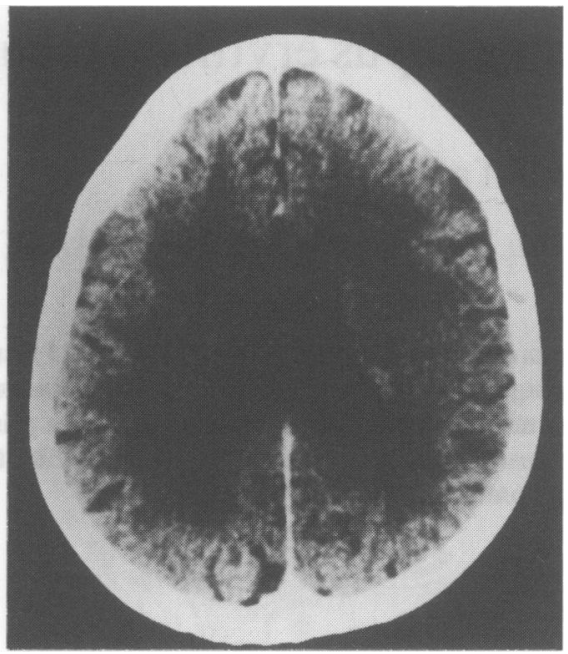

Figure 1 (a) CT brain scan showing enhancing lesions in the right cerebral hemisphere. (b) CT scan showing improvement after irradiation.

\section{Discussion}

The association of SLE and lymphoma is well recognized. ${ }^{1}$ Usually the lymphoma post-dates the diagnosis of SLE. However, rarely the connective tissue disease can occur many years after the diagnosis of lymphoma. ${ }^{2}$ The majority of the associated lymphomas occur in the peripheral lymph nodes. Other, rarer, sites include the lung and intestine. Previous reports of SLE and lymphoma with a similar tumour histology to our case describe only three cases. ${ }^{3,4}$ Only one was a primary cerebral lymphoma. Therefore both the tumour site and histological type were unusual in our patient.

It may be expected that the central nervous system is

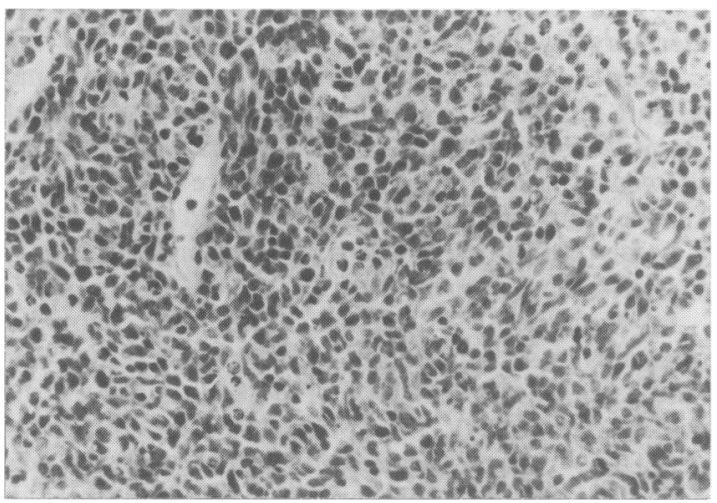

Figure 2 High power photomicrograph showing lymphoma histology (magnification $\times 400)$. especially susceptible to neoplasia because of a relative lack of immunosurveillance. This idea is compatible $\overrightarrow{0}$ with a series of immunosuppressed renal transplaq ${ }^{\infty}$ recipients; over half of those developing neoplasnisi had 'reticulum cell sarcoma' of the brain.' This is $\rightarrow$ a surprising contrast to the distribution of lymphoma in SLE.

A series of CT brain scans in 14 patients with SLE $\stackrel{\circ}{\circ}$ have been reviewed. ${ }^{6}$ There was evidence of infarction, $\stackrel{\varrho}{\Rightarrow}$ atrophy and haematomas in these patients. However, in most cases biopsy data were not reported, although one histologically proven meningioma was found.

Toxoplasmosis can occur in immunocompromised hosts including those with lymphoma ${ }^{7}$ and patients with SLE. ${ }^{8}$ Space occupying lesions of the brain can be caused by this infection. Serology may not be positive 3 . in all cases making diagnosis more difficult. ${ }^{7}$ In our patient biopsy was necessary for the final diagnosis of the lymphoma although we can not completely ruleo out a coexisting toxoplasma infection.

\section{Acknowledgements}

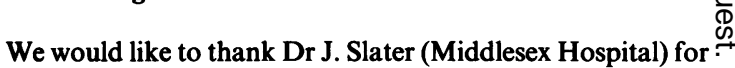
allowing us to present his patient, and Dr M. Bennett (Mount $\square$ Vernon Hospital) for histological interpretation and $\underset{\mathbb{D}}{\circ}$ photomicrographs. 


\section{References}

1. Green, J.A., Dawson, A.A. \& Walker, W. Systemic lupus erythematosus and lymphoma. Lancet 1978, ii: 753-756.

2. Berliner, S., Sidi, Y., Mar, C. et al. Systemic lupus erythematosus six years following chemotherapy for malignant lymphoma. Scand J Rheumatol 1985, 14: 276-278.

3. Hehir, M.E., Sewell, J.R. \& Hughes, G.R. Reticulum cell sarcoma in azathioprine treated systemic lupus erythematosus. Ann Rheum Dis 1979, 38: 94-95.

4. Lipsmeyer, E.A. Development of cerebral lymphoma in a patient with systemic lupus erythematosus treated with immunosuppression. Arthritis Rheum 1972, 15: 183-186.

5. Schreck, S. Cerebral neoplasms in renal transplants. Arch Neurol 1970, 22: 226-233.
6. Bilanuik, L.T., Suresh-Patel, M.B. \& Zimmerman, R.A. Computerised tomography of systemic lupus erythematosus. Radiology 1977, 124: 119-121.

7. Hakes, T.B. \& Armstrong, D. Toxoplasmosis; problems in diagnosis and management. Cancer 1983, 52: 15331540.

8. Dubin, H.V., Courter, M.H. \& Harrell, E.R. Toxoplasmosis, a complication of corticosteroid and cyclophosphamide treated lupus erythematosus. Arch Dermatol 1971, 104: 547-550. 